\title{
Iniquidades Sociais e Saúde Mental no Meio Rural
}

\author{
Magda Dimenstein - Universidade Federal do Rio Grande do Norte, Natal, Brasil \\ João Paulo Sales Macedo - Universidade Federal do Piani, Parnaíba, Brasil \\ Jader Leite - Universidade Federal do Rio Grande do Norte, Natal, Brasil \\ Candida Dantas - Universidade Federal do Rio Grande do Norte, Natal, Brasil \\ Monique Pfeifer Rodrigues da Silva - Universidade Federal do Rio Grande do Norte, Natal, Brasil
}

\begin{abstract}
Resumo
Esse artigo objetiva discutir casos de comorbidade de transtornos mentais comuns e uso abusivo de álcool e suas determinações entre moradores de assentamentos de reforma agrária. Trata-se de um estudo de delineamento combinado quantitativo-qualitativo, com amostra inicial de 2.012 moradores, resultando, após aplicação de questionário sociodemográfico e ferramentas de rastreamento (SRQ-20 e AUDIT) na identificação de 39 casos de comorbidade, aos quais foi aplicada entrevista semiestruturada. Para análise descritiva dos dados quantitativos utilizou-se o software SPSS for Windows, versão 20 e a construção de mapas dialógicos para os qualitativos. Aspectos envolvendo educação e trabalho, ambientes de interação/coesão social (redes e apoio social), mobilidade e transporte, acesso a equipamentos e serviços, falta de espaços de lazer, são fatores que interferem na saúde mental. Considera-se, dessa forma, a interdependência entre condições socioeconômicas, características dos territórios, padrões culturais, histórias de vida dos indivíduos e os agravos em saúde e morbidades psiquiátricas, em particular.

Palavras-chave: saúde mental, condições de vida, contextos rurais, vulnerabilidade
\end{abstract}

\section{Social Inequality and Mental Health in Rural Areas}

\begin{abstract}
The vulnerable psychosocial conditions found within poverty are made worse when dealing with populations from rural areas. These groups suffer the most from a lack of basic infrastructure and the problems that arise from the lack of social development. These people are hit the hardest, but remain less visible and far out of reach of public policies, especially in the case of mental health. For this discussion, we analyze cases of comorbidity of common mental disorders and alcohol abuse and its determinations among residents of land reform settlements. It is a combined quantitative-qualitative delineated study, with an initial sample of 2,012 residents. After the administration of a sociodemographic questionnaire and screening tools (SRQ-20 e Audit), 39 cases of comorbidity were identified, to which semi-structured interviews were administered. For descriptive analysis of the quantitative data, we used SPSS for Windows version 20, and the construction of dialogic maps for qualitative data. We observed the interdependence between socioeconomic conditions, the characteristics of territories, cultural patterns, individual life stories and mental diseases in particular.

Keywords: mental health, living standards, rural settings, vulnerability
\end{abstract}

\section{Inequidades Sociales y Salud Mental en Contexto Rural}

\section{Resumen}

El objetivo de este artículo fue discutir casos de comorbilidad de trastornos mentales comunes y uso exagerado de alcohol y sus determinaciones entre vecinos de la reforma agraria.Se trata de un estudio de delineamiento combinado cuantitativo cualitativo,con una muestra inicial de 2012 participantes,resultando después de aplicarse un cuestionario sociodemógrafico y herramientas de rastreamiento (SRQ-20 y AUDIT), la identificación de 39 casos de comorbilidad, a los cuales les fue aplicada una entrevista semiestructurada.Para el análisis descriptivo de los datos cuantitativos se utilizó el software for Windows, versión 20 y la construcción de mapas dialógicos para los datos cualitativos. Aspectos relativos a educación y trabajo, ambientes de interacción/cohesión social (redes y apoyo social) movilidad y transporte,acceso a equipamientos y servicios, falta de espacios de recreación, son factores que interfieren en la salud mental.Por lo tanto se considera la interdependencia entre condiciones socieconómicas, características de los territorios, padrones culturales, historia de vida de los individuos, y los agravios en salud y morbilidad psiquiátricas, en particular.

Palabras-clave: salud mental,condiciones de vida, contextos rurales, vulnerabilidad

\section{Introdução}

Esse artigo é um recorte de uma ampla investigação ${ }^{1}$ realizada junto a moradores de assentamentos

\footnotetext{
${ }^{1}$ Trata-se da pesquisa "Condições de vida e saúde mental em assentamentos rurais no nordeste brasileiro: diagnóstico, estratégias de

Disponivel em wnw.scielo.br
}

rurais no nordeste brasileiro, cujo objetivo foi mapear a incidência de transtornos mentais comuns (TMC) e os padrões de uso de álcool nessa população. Buscou-se

cuidado e suporte na rede de atenção primária e psicossocial". Foi um estudo com delineamento combinado (quantitativo e qualitativo), financiado pela Chamada Pública Universal 14/2012 do Conselho Nacional de Desenvolvimento Científico e Tecnológico/CNPq. 
compreender os processos sociais e subjetivos que constituem e caracterizam a vida nos assentamentos rurais e sua relação com a determinação social do processo saúde-doença-cuidado. Como ponto de ancoragem e discussão deste artigo, elegeu-se os casos detectados na amostra geral do estudo que apresentaram a peculiaridade de estarem situados acima do ponto de corte simultaneamente nos instrumentos de rastreio para TMC e detecção de padrão de uso de álcool, indicando a presença de casos com consumo de risco, uso nocivo e dependência de álcool e de sofrimento psíquico combinados, em potencialização recíproca ou comorbidade.

A saúde mental está no cerne do debate acerca das desigualdades e iniquidades em saúde uma vez que os transtornos mentais em geral apresentam atualmente uma das maiores cargas de morbidade, impactando de forma significativa o cotidiano dos indivíduos e familiares, culminando em dificuldades sociais e econômicas na sociedade como um todo. Além disso, há uma relação desproporcional entre quem está em sofrimento mental e quem recebe cuidados e tratamento (Organização Mundial da Saúde, 2009). De forma mais específica, os estudos têm aproximado a prevalência de TMC e o uso abusivo de álcool às condições socioeconômicas de determinados grupos humanos (Aquino, Nicolau, \& Pinheiro, 2011; Portugal, Corrêa, \& Siqueira, 2010; Vidal et al., 2014).

"Transtornos mentais comuns" é uma terminologia vastamente utilizada na literatura da epidemiologia psiquiátrica para a caracterização de quadros sintomáticos não psicóticos e sem patologia orgânica associada. Embora não preencham os critérios formais para diagnóstico de depressão e/ou ansiedade, segundo as classificações do DSM-IV e do CID-10 (Maragno, Goldbaum, Gianini, Novaes, \& César, 2006), os TMC reúnem sintomas depressivos e psicossomáticos, tais como insônia, fadiga, dificuldade de concentração que, além de causarem intenso sofrimento psíquico, geram incapacidade funcional comparável ou até pior aos quadros crônicos já estabelecidos (Carlotto, Amazarray, Chinazzo, \& Taborda, 2011; Costa \& Ludermir, 2005; Pinho \& Araújo, 2012). Quanto ao álcool, trata-se da substância mais consumida e acessível em todo o mundo na atualidade. $\mathrm{O}$ alto consumo é reconhecido como um problema de saúde pública por desencadear inúmeros prejuízos à saúde e demais dimensões da vida (social, familiar, econômico, laboral, etc.). Em âmbito global, o consumo de álcool tem aumentado consideravelmente nas últimas décadas, com predominância em países em desenvolvimento e que contam com pouca presença de políticas públicas voltadas para esse problema (Organização Mundial da Saúde, 2014).

No que tange ao abuso de substâncias entre portadores de transtornos mentais, trata-se de problemática frequente, cuja incidência vem aumentando consideravelmente, as quais geram enormes desafios não só em termos de avaliação e diagnóstico diferencial, mas também do ponto de vista dos cuidados necessários, especialmente pelos sintomas produzidos pelo uso de álcool, como ansiedade, depressão, agitação e os transtornos de conduta (Portugal, Corrêa, \& Siqueira, 2010). Nesses casos, os recursos farmacológicos precisam ser combinados, as estratégias terapêuticas diversificadas, os cuidados operados de forma continuada na rede de atenção psicossocial, enfim, há necessidade de uma atenção integral já que as taxas de recaída são maiores e há maior tendência ao abandono dos tratamentos. Nos casos onde há comorbidade, para além dos danos físicos e psiquiátricos já bastante conhecidos relacionados ao consumo problemático de álcool (enfermidades e lesões do tipo neoplásicas, infecções por HIV/AIDS, doenças hepáticas, síndromes de alcoolismo fetal, traumatismos por acidentes de trânsito e laborais, transtorno mentais (Organización Panamericana de la Salud, 2015), associam-se os de natureza psicossociais, como violência doméstica, desemprego, crises matrimoniais, conflitos familiares, bem como as repercussões produzidas do ponto de vista da inserção comunitária e apoio social, pouco abordados na literatura (OPAS, 2015).

A interdependência entre condições socioeconômicas, características dos territórios e dos ambientes vividos, padrões culturais, experiências e histórias de vida dos indivíduos e os impactos negativos na saúde mental, especificamente no que diz respeito aos TMC e uso problemático de álcool, está, portanto, na centralidade da argumentação proposta nesse artigo, a qual está sustentada na perspectiva da Determinação Social da Saúde (Antunes, 2015; Costa, Dimenstein, \& Leite, 2014; Ferreira, Sales, Casotti, Bispo Júnior, \& Braga Júnior, 2011). Essa complexa e dinâmica relação não pode ser compreendida de forma determinista, mas como singularidades não passíveis de generalizações.

Os casos de comorbidade (TMC e uso problemático de álcool), que são alvo de discussão neste artigo, compartilham e concentram um conjunto de elementos detectados no cotidiano dos assentamentos rurais, bem como nas histórias de vida dos moradores, que parecem potencializados nesses casos específicos, revelando as articulações entre condições de vida, vulnerabilidades 
psicossociais e ambientais, suporte psicossocial, modos de subjetivação, formas de adoecimento e enfrentamento das adversidades no meio rural. Em função desse conjunto de aspectos, essas situações foram denominadas de "casos-problema".

Nesse sentido, considera-se fundamental para a compreensão dessas situações o reconhecimento de uma perspectiva de saúde mental integral, em que se faz necessário superar abordagens ancoradas na determinação única de fenômenos multidimensionais; trabalhar com a ideia de individual enquanto relação ou intersubjetividade, do social como contexto de interação ou "espaços de experiência concreta da intersubjetividade", e com as "relações sociais que estão na base das situações de vulnerabilidade e de negligência ou violação dos direitos humanos" (Ayres, Paiva, \& Buchalla, 2012, p.13).

Alinha-se a essa perspectiva a abordagem multidimensional da pobreza que, para além da insuficiência de renda, incorpora outras dimensões que ampliam sobremaneira a compreensão dos modos de vidas e das implicações psicossociais da pobreza. Diz respeito ao não acesso a bens, produtos, políticas, sendo um fenômeno complexo relacionado à exclusão social, indigência, desfiliação, fragilização de laços sociais e pertencimento, violência, destruição ambiental, solidão, injustiça, aspectos que repercutem nos modos de subjetivação, na reprodução intergeracional das condições de vida e na mobilidade social.

Assim entendido, o conceito de pobreza passa a incorporar privações em diversos âmbitos (além do monetário), com desdobramentos subjetivos (Cidade, Moura Jr., \& Ximenes, 2012; Sawaia, 2009), repercutindo nas esferas dos direitos básicos, como apontam Crespo e Gurovitz (2002) em revisão sobre as contribuições de Sen e Deepa Narayan:

Há a falta do que é necessário para o bem-estar material. Associa-se a esse conceito a falta de voz, poder e independência dos pobres que os sujeita à exploração; à propensão à doença; à falta de infraestrutura básica, à falta de ativos físicos, humanos, sociais e ambientais e à maior vulnerabilidade e exposição ao risco (p. 11).

Nas últimas décadas, é inegável a melhora dos indicadores sociais do país, especialmente nas zonas rurais, como a queda nos índices de desigualdade, de pobreza e extrema pobreza ${ }^{2}$, crescimento da renda

${ }^{2}$ Os quatro estratos definidos a partir da renda domiciliar per capita adotados como linhas de pobreza pelo Programa Bolsa Família e o

Psico-USF, Bragança Paulista, v. 22, n. 3, p. 541-553, set. / dez. 2017 per capita, bem como naqueles relativos à moradia, educação, saúde e lazer. Entretanto, a situação ainda é alarmante quando se considera, por exemplo, que um em cada quatro brasileiros que vivem no campo está em situação de pobreza extrema (Mello, 2015).

Assim, ainda se observa nesses espaços a manutenção da migração rural-urbano em função das dificuldades de sobrevivência no campo, o desenvolvimento de atividades ocupacionais informais complementares ao trabalho agrícola e aumento da dependência dos programas sociais e de transferência de renda, especialmente pelos extremamente pobres. Ademais, registra-se avanço insuficiente quanto ao acesso à infraestrutura (abastecimento de água pela rede pública, coleta de lixo, escoamento sanitário adequado e energia elétrica) e às políticas públicas de saúde e educação.

Oliveira, Cazella, Tecchio e Mireski (2015), analisando as ações das políticas de desenvolvimento territorial para superação da pobreza rural, identificaram inúmeros problemas dentre os quais a exclusão de boa parte da população que deveria ser beneficiada e a falta de planejamento consistente com financiamento inadequado de várias ações. Sobre esse cenário, sabe-se, de acordo com Schneider (2010), que

\begin{abstract}
As condicões e possibilidades do desenvolvimento rural e a alteração das condições de vida das populações em situação de vulnerabilidade esbarra em mecanismos de dominação social e cultural que são construidos historicamente por elites locais para legitimar seu poder e manter os privilégios que sustentam suas posições (p. 522).
\end{abstract}

Isso leva o autor a concluir que a tradição política de tipo clientelista e patriarcal obstaculizaria não só as transformações estruturais, mas, fundamentalmente, as mudanças de natureza sociocultural no meio rural do Brasil. Essa situação, além de mais fortemente encontrada no meio rural, é também predominante na região Nordeste, onde há mais concentração de pobres e extremamente pobres, reunindo 49,75\% das famílias beneficiadas pelo Programa Bolsa Família em todo o país, principal estratégia de enfrentamento à pobreza adotada pelos governos brasileiros nos últimos anos (Brasil, 2013).

Plano Brasil Sem Miséria consideravam, no ano de 2011, “extremamente pobres" as famílias com renda inferior a R $\$ 70,00$; "pobres" aquelas com renda maior ou igual a $\mathrm{R} \$ 70,00$ e inferior a $\mathrm{R} \$ 140,00$; "vulneráveis" as que possuem renda maior ou igual a $\mathrm{R} \$ 140,00$ e menor que R \$ 560,00 e "não pobres" aquelas com renda maior ou igual a R\$560,00 (Souza \& Osório, 2013). 
É inegável o papel positivo que tais ações têm hoje no cotidiano das famílias brasileiras em situação de pobreza. Em um cenário em que historicamente muito pouco esforço foi empreendido no sentido de propiciar melhores condições de vida aos pobres brasileiros, especialmente no meio rural, em que pese tratar-se de uma medida compensatória que não redistribui renda e que, portanto, não altera o perfil de concentração de renda brasileira, as ações de enfrentamento à pobreza adotadas são um avanço político e absolutamente indispensáveis no momento atual, não havendo, ainda, alternativas viáveis no horizonte político brasileiro.

Mesmo com os programas Bolsa Família e o Plano Brasil sem Miséria, implantados no Governo do Partido dos Trabalhadores, a partir de 2004, ambos orientados para melhoria dos indicadores de pobreza no país, apoiados em uma concepção multidimensional, com pautas específicas para o espaço rural e as populações de assentamentos, tendo como meta articular as políticas de desenvolvimento social com ações de inclusão produtiva rural (Mello, 2015), os níveis de pobreza nas populações do campo são abissais. Para Mauriel (2010) e Siqueira (2013), o que houve foi a melhoria de indicadores para patamares aceitáveis de pobreza, na perspectiva do seu gerenciamento e não da sua superação.

Posto isso, estudos como de Ottonelli e Mariano (2014), ao mensurar a pobreza multidimensional ${ }^{3}$ nos municípios da região Nordeste focando nas dimensões relativas à educação, saúde, condições habitacionais e renda, reforça a posição de que a pobreza significa privação em diferentes dimensões relacionadas com a vida das pessoas e aponta que a pobreza na região Nordeste é um problema complexo com características multidimensionais.

Indicadores socioeconômicos revelam que os espaços rurais, especialmente os da região Nordeste, ainda apresentam precárias condições de vida: baixo nível financeiro, desemprego; alto índice de não alfabetizados, de insegurança alimentar e mortalidade infantil para os padrões atuais; dificuldade no acesso aos serviços públicos e assistência técnica; precariedade das condições de trabalho e maior dependência dos programas de transferência de renda e falta de apoio social (Camargo, Curralero, Licio, \& Mostafa, 2013; Instituto Nacional de Colonização e Reforma Agrária, 2010). É sobre as populações do campo que recai a maior parte dos efeitos da falta de infraestrutura

\footnotetext{
${ }^{3}$ Estudo fez uso de 19 indicadores distribuídos em quatro dimensões: educação, saúde, condições habitacionais e renda por meio da teoria dos conjuntos furzy (Ottonelli \& Mariano, 2014).
}

básica e dos problemas típicos da falta de desenvolvimento social. Esses grupos são mais afetados, menos visíveis e mais difíceis de alcance pelas políticas públicas e investigadores.

Essas condições de desvantagem social aumentam a vulnerabilidade das populações rurais ao sofrimento psíquico, em especial aos TMC e uso problemático de álcool. Vários estudos reforçam a inter-relação entre condições precarizadas de vida e saúde mental, incorporando elementos que fazem parte do cotidiano desses moradores, como a violência, as questões de gênero e as modalidades de apoio social (Costa et al., 2014; Loureiro, Lima, Partidário, \& Santana, 2015; Moura, Ximenes, \& Sarrieira, 2014; Remoaldo \& Nogueira, 2013; Silva \& Menezes, 2014).

Camurça, Alencar, Cidade e Ximenes (2016) destacam as implicações psicossociais da seca em regiões do Nordeste, as quais tendem a produzir insegurança quanto ao futuro, sentimentos de desânimo e tristeza, fatalismo e desesperança, que impactam nos modos de vida e nos processos de saúde dessa população na medida em que afetam os ideais que elaboram sobre si e sobre o mundo, bem como suas estratégias de enfrentamento das adversidades.

Levando-se em consideração os tempos de crise econômica, com seus desdobramentos no aprofundamento das situações de desemprego, pobreza, endividamento ou perda da capacidade socioeconômica inclusive das populações do campo, percebe-se a associação desses fatores ao surgimento de sofrimento psíquico e/ou agravamento de quadros de transtornos mentais, com destaque para depressão, ansiedade, suicídio e consumo de álcool e outras drogas (Antunes, 2015; Silva, Cardoso, Saraceno, \& Almeida, 2015).

O ponto crucial a destacar é que as condições de vulnerabilidade psicossocial relativas à pobreza se agravam quando se trata das populações rurais. Desigualdade, pobreza e enfermidade constituem, portanto, um típico movimento de retroalimentação (Cotta et al., 2007). Isso afeta a vida concreta das pessoas e tem implicações subjetivas importantes, atingindo a dimensão política da existência humana, com reverberações nos processos de descoletivização e privatização do cotidiano.

Pussetti e Brazzabeni (2011) falam de sofrimento social, isto é, de um sofrimento que tem "as suas raízes nas fraturas devastantes que as forças sociais podem exercitar sobre a experiência humana, onde uma certa configuração da ordem social restringe a capacidade e a liberdade de escolha de certos 
indivíduos ou grupos” (p. 469). Em outras palavras, existem relações profundas entre a experiência subjetiva do mal-estar e os processos históricos e sociais mais amplos, que precisam ser considerados pela epidemiologia psiquiátrica.

A realidade dos assentamentos potiguares e piauienses pesquisados segue a tendência acima apresentada, a qual favorece o aparecimento de inúmeros problemas quanto ao processo saúde-doença-cuidado. Os "casos-problema", indicadores de comorbidade do uso abusivo de álcool e de sofrimento psíquico, em potencialização recíproca, aqui abordados, são emblemáticos quando se trata dessa inter-relação de fatores. É sobre eles que se vai tratar a seguir.

\section{Método}

\section{Participantes}

A amostra da pesquisa geral foi de 2.012 moradores, sendo 762 homens e 1.250 mulheres moradores de nove assentamentos no Estado do Rio Grande do Norte e seis no Estado do Piauí. Trata-se de uma amostra censitária. O perfil dos moradores é de pessoas com idade entre 20 a 49 anos $(M=42,44 ; D P=16,41)$, casadas $(48,87 \%)$, com ensino fundamental completo/ incompleto $(58,19 \%)$, cuja ocupações são prioritariamente de estudante $(29,65 \%)$ e agricultor $(26,80 \%)$, com renda familiar de até $1 / 2$ salário mínimo (32,51\%). Do total de moradores, 228 (11,3\%) ficaram acima do ponto de corte no instrumento de rastreamento do uso problemático de álcool e 315 (15,6\%) de TMC. De forma mais específica, 39 casos pontuaram simultaneamente nos dois instrumentos acima do ponto de corte. A discussão proposta neste artigo está focada nesses casos específicos ou "casos-problema", tal como anteriormente indicado.

\section{Instrumentos}

\section{a) Questionário sociodemográfico-ambiental} (QSDA), utilizado para auxiliar na identificação do número de famílias residentes nos assentamentos e informações sobre as condições de vida, saúde e trabalho das famílias assentadas. Trata-se de uma versão modificada do material elaborado pelo Departamento de Geologia da UFRN, configurando-se num questionário misto (questões abertas e fechadas) e múltiplos formatos (escolha múltipla simples, dicotômica simples e itens em checklist). Os itens abordaram os seguintes aspectos: renda, fonte de sustentação, ocupação, escolaridade, número de moradores residentes, idade, sexo, religião, infraestrutura dos assentamentos, condições de saúde, acesso aos serviços públicos de saúde, bem como aos programas de transferência de renda, crédito e assistência rurais, participação e organização política.

b) Self-Reporting Questionnaire (SRQ-20) como ferramenta rastreadora de transtorno mental comum (TMC). O SRQ-20 foi validado e recomendado pela OMS para estudos comunitários e na atenção primária à saúde, principalmente nos países em desenvolvimento, sendo validado para o Brasil por Mari e Williams (1986). É composto por 20 itens, sendo quatro sobre sintomas físicos, e dezesseis sobre sintomas emocionais, com duas possibilidades de resposta (sim/não). Os escores obtidos sinalizam a probabilidade de presença de TMC ou desconforto emocional (construto avaliado), variando de 0 (nenhuma probabilidade) a 20 (extrema probabilidade). Não inclui questões sobre sintomas psicóticos nem sobre o consumo de álcool e outras drogas. Nessa pesquisa, foi adotado o ponto de corte de 07 ou mais respostas positivas como indicativo de TMC.

c) Alcohol Use Disorders Identification Test (AUDIT) como ferramenta rastreadora inicial de uso de álcool (consumo de risco, uso nocivo e dependência) nos serviços de atenção primária e apoio para intervenções breves pelos profissionais. Contêm dez itens, cada um com margem de 0 a 4 pontos, resultando no espectro de pontuação de 0 a 40, com a seguinte classificação: Zona I (baixo risco) - 0 a 7 pontos; Zona II (uso de risco) - 8 a 15 pontos; Zona III (uso nocivo) - 16 a 19 pontos; Zona IV (provável dependência) - 20 a 40 pontos. O ponto de corte adotado foi 08 ou mais itens, com respostas positivas para consumo de risco, nocivo ou provável dependência.

Para os que atingiram o ponto de corte nas ferramentas de rastreamento, foram realizadas entrevistas semiestruturadas, seguindo roteiro previamente elaborado em torno dos seguintes aspectos: histórico, frequência e modos de uso do álcool; histórico e problemas relacionados ao bem-estar emocional; dinâmica familiar; contexto comunitário; características do trabalho; condições de vida e dispositivos formais e informais de saúde utilizados. Todos os instrumentos foram aplicados de 
forma individual, na residência do morador, com tempo médio de 2 horas para as 3 ferramentas.

\section{Análise dos Dados}

Para análise dos dados referentes aos resultados do QSDA, SRQ-20 e AUDIT, utilizou-se o software Statistical Package for the Social Sciences (SPSS) for Windows, versão 20. A partir do SPSS, realizou-se análise descritiva dos dados, onde foi utilizada frequência absoluta, relativa e mediana (valor mínimo e máximo), bem como o teste do $\chi^{2}$ que se destina a encontrar um valor da dispersão para duas variáveis nominais, avaliando a associação existente entre variáveis qualitativas. Em relação à análise das entrevistas, utilizou-se mapas dialógicos para compreender os significados atribuídos à problemática suscitada pela entrevista e estabelecer conexões entre as falas dos participantes, observações dos pesquisadores e a literatura de referência (Spink, Brigadão, Nascimento, \& Cordeiro, 2014).

Foram cumpridos os seguintes passos para elaboração dos mapas dialógicos: 1) transcrição integral das entrevistas; 2) categorização dos conteúdos de acordo com os eixos de análise; 3) discussão coletiva acerca das categorias sínteses de análise (reconhecimento do problema, fator disparador, tipo de recursos formais e informais utilizados, apoio social, dentre outros; 4) elaboração final dos mapas referentes ao resultado do AUDIT e SRQ-20. Por fim, foram cumpridos os procedimentos éticos da Resolução do Conselho Nacional de Saúde de $\mathrm{n}^{\circ}$ 466/12 e todos os participantes assinaram Termo de Consentimento Livre e Esclarecido.

\section{Resultados e Discussão}

\section{Etapa Quantitativa: TMC e Padrão de Uso de Álcool em Assentamentos de Reforma Agrária}

\section{a) Perfil Sociodemográfico dos "Casos-Problema"}

Dos 39 casos que pontuaram simultaneamente no SRQ-20 e no AUDIT, indicando incidência de TMC e uso problemático de álcool (denominados como "casos-problema"), 23 são do Rio Grande do Norte e 16 do Piauí. Houve uma distribuição equilibrada entre homens (21) e mulheres (18) quando analisada a divisão por sexo agregando os dois estados. Esses sujeitos são majoritariamente casados, agricultores e donas de casa, com grau de escolaridade no nível de Ensino Fundamental (completo/incompleto - até 4 anos de estudo) e faixa etária entre 18 e 40 anos $(M=40,36$; $D P=18,28)$. Grande parte das famílias é beneficiada pelo Programa Bolsa Família, têm renda familiar de $1 / 2$ a 1 salário mínimo (U\$200,00), sendo pouco expressiva a parcela que recebe auxílios do Programa Nacional de Fortalecimento da Agricultura Familiar (PRONAF) e de assistência técnica (fornecida pela empresa/instituto de Assistência Técnica e Extensão Rural - EMATER).

Esses 39 moradores estão concentrados em alguns assentamentos que têm em comum o fato de apresentarem certos condicionantes que estão para além de características regionais ou locais, os quais são recorrentes no cotidiano de vida dos moradores do meio rural brasileiro, e que estão associados aos resultados encontrados em termos da incidência de transtornos mentais comuns e uso problemático de álcool. Destaca-se o considerável número de moradores não alfabetizados, a dificuldade de acesso como uma das fortes características geográficas desses assentamentos (o que também limita ou dificulta alcance mínimo ou satisfatório aos serviços de saúde e assistência social), bem como uma frágil mobilização comunitária e apoio social, aspectos fortemente destacados nas entrevistas com seus moradores e lideranças.

\section{b) Caracterização em Relação aos Itens Pontuados do SRQ-20}

Observou-se que todos os 39 casos pontuaram nas quatro dimensões da ferramenta (humor depressivo-ansioso; decréscimo da energia vital; sintomas somáticos; pensamentos depressivos), três a quatro vezes a mais em todos os itens do instrumento se comparados aos resultados da amostra situada acima do ponto de corte. Esses resultados são preocupantes e revelam o nível de sofrimento de homens e mulheres que parecem mais sensíveis às características ambientais, sociais e culturais de seus territórios, e se mostram mais vulneráveis aos problemas de depressão e ansiedade, bem como ao consumo excessivo de álcool e declínio dos níveis de saúde de uma forma geral. Além disso, alertam para o típico movimento de retroalimentação que articula pobreza, desigualdade e saúde mental (Antunes, 2015; Guimarães et al., 2011).

c) Caracterização em Relação aos Itens Pontuados do AUDIT

Os escores obtidos no AUDIT dos casos-problema reproduzem o mesmo padrão de consumo de álcool no resultado geral da pesquisa, com maior registro de pontuações na zona de consumo de risco, concentrando 28 dos 39 casos $(71,79 \%)$ e na qual também pontua grande parte das mulheres (46,15\%). Entretanto, é importante 
enfatizar que nas análises estatísticas foi detectado que os "casos-problema" apresentaram escores mais altos se comparados aos resultados obtidos no AUDIT pelo restante da amostra, pontuando três a quatro vezes mais em todos os quesitos do instrumento e de cinco a dez vezes mais quando se trata das categorias uso nocivo e dependência, revelando um padrão mais problemático de consumo de álcool que o restante da amostra. Os resultados referentes ao padrão de consumo dos "casos-problema" ilustram a articulação entre fatores socioculturais e pessoais que influenciam no aumento do consumo de álcool nesses territórios. As consequências desse padrão de consumo, tanto socioeconômicas quanto para a saúde, são indiscutíveis.

Em outras palavras, os assentamentos que concentram os 39 casos-problema revelam características do contexto que influenciam a saúde mental, em particular na incidência de transtornos mentais comuns e consumo abusivo de álcool: as do ambiente físico e construído (mobilidade e transporte; acesso a equipamentos e serviços; falta de espaços de lazer); as do ambiente socioeconômico (educação, trabalho) e as do ambiente de interação e coesão social (redes e apoio social) (Loureiro et al., 2015). Indicam assim que a saúde mental está articulada às características dos territórios e ambientes vividos, às relações interpessoais, ao cotidiano marcado pelo isolamento físico e social, reforçando os achados de Ottonelli e Mariano (2014) sobre as condições de vida na região Nordeste que estão associadas aos agravos em saúde e morbidades psiquiátricas, em particular.

\section{Etapa Qualitativa: Pobreza, Desigualdades e Saúde Mental}

$\mathrm{Na}$ etapa qualitativa do estudo, as entrevistas realizadas com os 39 participantes aportaram informações que ajudam na compreensão desse cenário preocupante em termos de saúde mental. Buscou-se conhecer a perspectiva dos sujeitos diante dos resultados da pesquisa, suas histórias em torno do uso do álcool e do sofrimento mental, assim como sua avaliação acerca do contexto de vida nos assentamentos.

O conceito de determinação social da saúde é o elemento articulador das análises e traz como pressuposto fundamental uma compreensão ampliada da saúde por meio de um olhar interdisciplinar sobre as formas de organização da sociedade, de sua estrutura social e econômica, entendendo que a produção da saúde, da doença e do cuidado está diretamente a elas associada. Nesse sentido, não envolve apenas indicadores de desigualdade social e pobreza, mas contempla questões como a presença, qualidade e acessibilidade aos serviços e ações de saúde pública e sua interface com outras políticas setoriais, aos recursos comunitários, bem como compreende como as vizinhanças se configuram, o grau de integração e suporte social presente em uma comunidade.

Com base nessa perspectiva, foi possível observar que inúmeros fatores estão associados à incidência de TMC e uso problemático de álcool entre os moradores rurais: eventos de vida estressantes; perdas e rompimentos afetivos; problemas familiares, conjugais e de saúde; baixa escolarização; dificuldades financeiras; violência; histórico de uso de álcool e de transtornos mentais na família; contexto situacional e estimulador do consumo de álcool, com impacto especial entre os homens; além das formas de organização social e de desgaste do capital social com o enfraquecimento das relações de solidariedade e apoio social nos contextos dos assentamentos.

As dimensões que mais se destacaram nas narrativas foram as questões de gênero, as condições de trabalho, a renda no cotidiano das famílias, a seca, a relação com os serviços de saúde, os modos de convivência comunitários e as dinâmicas familiares, temas que atravessam as narrativas e compõem um cenário de estreita relação com disparadores do sofrimento e com as possibilidades e estratégias para lidar com o mesmo.

Nas entrevistas relativas ao uso de álcool, constatou-se que as histórias têm em comum o uso de bebidas destiladas e fermentadas, especialmente cachaça e cerveja, que são consumidas com familiares e amigos, geralmente em festas, em casa e em bares e com o objetivo de diversão, socialização e alívio de preocupações, decepções e outros sofrimentos. Grande parte desses moradores consome álcool desde a adolescência ou juventude (alguns casos remeteram à infância), sendo o uso caracterizado como "recreativo", o que muitas vezes faz com que não reconheçam o uso problemático da substância. Os fatores disparadores mais recorrentes na iniciação do consumo estão vinculados à necessidade de socialização, pertencimento e aprovação social.

Evidenciou-se que os assentamentos pesquisados se configuram como um contexto estimulador do consumo, já que existe muita facilidade em se obter bebidas alcoólicas nesses espaços, considerável número de consumidores habituais dentre os assentados, a presença de bares e festas com uma das poucas possibilidades de lazer e entretenimento nos assentamentos, indicando uma certa banalização do uso e naturalização dos problemas associados. 
Silva e Menezes (2014) apontam para elementos semelhantes em pesquisas com populações rurais, que vivem em contexto promotor de consumo: facilidade em se obter bebidas, uso generalizado nos mais diversos eventos de vida, inclusive associado à algumas atividades laborais, influência dos familiares e amigos. Essa realidade também foi registrada no relatório da OPS (2015) sobre as tendências do uso de álcool nas Américas e suas consequências para a saúde na região.

A maioria dos participantes alega nunca ter buscado ajuda nos serviços de saúde em função do consumo do álcool. Todos afirmam terem controle sobre o consumo, negam o uso frequente do álcool e destacam o trabalho como fator regulador. Somente quando há problemas graves em termos da saúde física (como necessidade de hospitalização, por exemplo), interferência nas atividades do cotidiano (família e trabalho) e impacto no orçamento familiar é que o álcool passa a ser reconhecido como um problema. Isso acontece mais frequentemente entre os homens do que entre as mulheres que, geralmente, não reconhecem o uso do álcool como problemático, indicando a interferência de questões culturais e de gênero.

Nesse sentido, observa-se que os participantes seguem tendência semelhante a que foi apontada na literatura quanto às diferenças de gênero no desempenho, permissividade e aceitação do consumo: homens fazem uso mais frequente e intenso que as mulheres (Ferreira et al., 2011), há mais estigma sobre as mulheres que fazem uso de álcool do que sobre os homens (Silva \& Menezes, 2014), os quais reconhecem mais facilmente o uso prejudicial do que as mulheres (OPS, 2015).

Algumas entrevistas trazem relatos acerca das motivações, modos de uso e consequências do consumo de bebida alcoólica na vida desses moradores. Nesses casos, há sobreposição de desassistência em saúde, isolamento social, situações de violência, de conflitos na família e na comunidade, gerando forte estigma em relação a esses moradores que fazem uso abusivo de álcool.

A qualidade do acolhimento, a resolutividade e capacitação técnica das equipes para lidar com a questão da dependência química são fontes de críticas. Os moradores indicam a necessidade de cuidado especializado, com sensibilidade e capacidade de enfrentar os problemas associados à pobreza e desigualdade social. Reconhecem que estão inseridos em um cenário extremamente estimulador e permissivo, seja pela facilidade de acesso, seja pelo consumo habitual generalizado principalmente entre amigos e membros da família, pela falta de opção de lazer e divertimento, pela possibilidade de enfrentamento dos problemas que não recebem retaguarda da comunidade e da família.

Em termos do resultado do SRQ-20, foi possível observar pelas entrevistas uma variedade de fatores associados aos TMC. Quanto ao indicativo de TMC, os participantes, diferentemente do uso problemático de álcool, reconhecem mais facilmente o resultado da pesquisa, porque sentem cotidianamente alteração de humor, medo, tristeza, ansiedade, solidão, queixam-se de variados sintomas físicos, como dores e fadiga, sentem-se isolados, incapazes de trabalhar, sem autonomia para tomar decisões, enfim, insatisfeitos com a vida. Alguns indicam histórico de transtornos mentais na família, o que facilita o reconhecimento de que algo não vai bem.

Como fatores disparadores, destacam-se quadros de vulnerabilidade, como o desemprego, as dificuldades financeiras e situações de violência. Perdas afetivas, rompimentos, assim como problemas familiares os mais diversificados foram aspectos detectados. Em uma perspectiva de interseção entre trabalho e gênero, foi relatado especialmente pelas mulheres, a sobrecarga pelo exercício de diferentes papéis sociais e cuidado com a família, estresse na relação conjugal, nascimento dos filhos e processo de envelhecimento. São recorrentes as situações de sofrimento entre os homens por ausência de autonomia/segurança financeira, pelo receio de perder a terra, de não poder trabalhar por motivo de doença e ainda os desafios na complementação de renda para o sustento mensal (dívidas e sobrecarga de trabalho) como desencadeadoras e agravantes de quadros de nervosismo e ansiedade.

Ou seja, homens e mulheres vivem cotidianamente suas atribuições de modo diferenciado e isso resulta em modos particulares de adoecimento. Os homens destacam o receio de não prover renda suficiente para o sustento da família e a importância de trabalhar na própria terra. Foi frequente o relato sobre a angústia e receio que têm em perder capacidades e oportunidades em protagonizar aquilo que Detoni (2010) ressalta como a "lógica do provedor" (p.72) em sua discussão sobre construção de subjetividade entre barrageiros, considerada uma questão central do trabalho como estrutura da sociedade capitalista e da construção do masculino.

Para as mulheres, a tentativa de conciliar diferentes atividades e responsabilidades, como o estudo, o cuidado e a responsabilização por parentes, a vida conjugal, o trabalho doméstico e na agricultura, bem como 
a precariedade de vínculos empregatícios e a ausência de autonomia financeira, são queixas disparadoras ou agravantes do sofrimento.

Esses elementos conectam-se diretamente com particularidades identificadas nos assentamentos como as dificuldades próprias do trabalho agrícola (em função da sazonalidade do plantio e colheita e dos períodos de estiagem severos, que dificultam a pesca, a lavoura e pecuária), a divisão sexual do trabalho com encargos consideráveis para as mulheres e insuficiência do sustento familiar apenas com proventos da agricultura, fazendo-se necessária a combinação de auxílios de programas sociais, prestação de serviços e aposentadoria.

Analisando, portanto, os 39 casos-problema a partir dos elementos em comum que permeiam os relatos dos participantes, observa-se que as dinâmicas familiares são disparadoras tanto para o consumo de álcool quanto para o sofrimento mental, a partir da vivência recorrente de perdas e rompimentos afetivos, de transtornos mentais entre familiares, histórico de uso de álcool na família, situações de violência e dificuldades socioeconômicas que afetam a todos. A vivência cotidiana de situações críticas e a sobrecarga das mulheres em combinar papéis de provedora, cuidadora da família e acompanhante do tratamento de parentes doentes são apontados também pelo II LENAD (Instituto Nacional de Ciência e Tecnologia para Políticas Públicas do Álcool e outras Drogas, 2012) como disparadores do uso de álcool abusivo no cotidiano familiar.

A violência também foi citada nas entrevistas como elemento disparador para sofrimento psíquico e consumo de álcool, especialmente por ser negligenciada no cotidiano. Alguns estudos destacam a vulnerabilidade de certos contextos onde há a presença de alcoolismo, violência e maior exposição ao sofrimento psíquico, em especial entre as mulheres (esposas) e filhos como principais vítimas (Lindner, Coelho, Bolsoni, Rojas, \& Boing, 2015; Miranda, Paula, \& Bordin, 2010; Pereira et al.,2015).

Relatos de preocupações com a insegurança econômica e as dívidas, deslocamento dos parentes para outras regiões do país em busca de emprego, bem como com as dificuldades financeiras que impedem a aquisição de bens materiais, alimentos, consultas médicas e remédios, são frequentes entre os moradores. Desse modo, as dinâmicas familiares são afetadas pelas condições socioeconômicas que produzem impactos negativos no dia a dia, como tristeza e insatisfação com a vida. Isso, associado à precariedade na oferta de profissionais, serviços de saúde e assistência social nesses territórios, produz aumento da vulnerabilidade e tem forte impacto na saúde mental.

O aumento de consumo de álcool e sofrimento mental em tempos e situações de crise econômica com aumento do desemprego, com o trabalho precarizado e a estruturação instável e competitiva das economias são aspectos relatados na literatura (Antunes, 2015). $\mathrm{Na}$ tentativa de compreender essa trama de fatores que afeta a vida de milhões de pessoas no mundo, considera-se que, em cenários desfavoráveis economicamente, há o recrudescimento de sentimento de insegurança, desproteção, incerteza, instabilidade, medo, os quais enfraquecem os fatores protetores da saúde mental e alteração do padrão de utilização dos serviços de saúde, com aumento considerável de consultas e internação psiquiátrica.

Identificou-se, nos assentamentos, falta de coesão social combinada com posturas indiferentes em relação aos problemas da comunidade e muita desconfiança em compartilhar problemas. Desse modo, recorre-se geralmente às formas individualizadas de enfrentamento, como as tentativas de autossuperação e ajuda divina, revelando a articulação entre as formações sociais contemporâneas perpassadas por lógicas individualizantes, vida cotidiana e sofrimento psíquico.

Nesse sentido, cada vez mais o sucesso e o fracasso são responsabilidades individuais, a desintegração das redes sociais de reconhecimento e proteção é uma evidência, assim como os espaços coletivos e comunitários vêm se desintegrando, gerando enorme desamparo. Tomando como base Cidade, Moura e Ximenes (2012) ressaltam que essa lógica facilita processos de esvaziamento da historicidade das condições de vida ao atribuir a uma dimensão "incontrolável" aos fatos (p. 94) e até mesmo, como dizem Ewald, Moura e Goulart (2012), a sensação de fraqueza/fracasso diante das expectativas de resolução individual do problema. Isso termina favorecendo o isolamento social e desestimula a busca por ajuda, especialmente pelos moradores que consomem álcool de modo problemático.

A configuração desses processos de adoecimento não encontra acolhida satisfatória nos serviços de saúde que têm responsabilidade de dar retaguarda aos assentamentos. A maioria dos participantes alega não ter procurado serviço de saúde, não somente pelas dificuldades de acesso, mas também por experiências de acolhimentos insatisfatórios/não resolutivos.

Geralmente não reconhecem a necessidade de cuidados em saúde mental frente a outros quadros mais importantes (as "doenças de verdade") merecedores 
de atenção pelas equipes, mas também têm receio do estigma que ainda perdura no campo da saúde mental (Maragno et al., 2006; Martins, 2012). Sinalizam a necessidade de diversificação profissional e inclusão do psicólogo na equipe, de mais equipamentos de saúde (como farmácias próximas aos assentamentos), bem como a implantação de grupos de ajuda, palestras explicativas, etc., para os casos de transtornos e uso problemático de álcool.

\section{Considerações Finais}

Buscou-se destacar neste trabalho que a pobreza é um fator decisivo e tem rebatimentos inequívocos na saúde mental, em especial no que tange à prevalência de transtornos mentais comuns e o uso abusivo de álcool. Ademais, que a pobreza agudiza as iniquidades sociais e em saúde no meio rural e acirra os processos de exclusão social das populações que aí vivem. Nesse sentido, os assentamentos e assentados rurais podem ser considerados contextos e populações vulneráveis que necessitam de sistemas de proteção e retaguarda que garantam a efetivação de direitos e cidadania.

Contudo, as inter-relações entre desigualdades sociais e o processo saúde-doença-cuidado são tramadas de forma complexa compondo um certo plano de organização ao tecer os fios das histórias de vida, das dinâmicas de funcionamento familiar, das formas de organização social, das características geográficas dos espaços, fios que vão moldando subjetividades e territórios existenciais, constituindo as singularidades dos processos de vida e a heterogeneidade das vivências de sofrimento psíquico.

Nesse sentido, é necessário ampliar as investigações com populações rurais, de modo que esse conjunto complexo e imbricado de determinações possa ser considerado tanto pelos formuladores de políticas públicas, quanto gestores e trabalhadores presentes no cotidiano dos serviços para que se possa ampliar as ações de cuidado, acompanhamento e monitoramento dos casos, inclusive com ações preventivas e de promoção em saúde, articuladas a outras bandeiras de luta dos assentados da reforma agrária, com vistas ao enfrentamento das iniquidades que condicionam a saúde mental.

\section{Referências}

Antunes, J. A. P. (2015). Crise económica, saúde e doença. Psicologia, Saúde \& Doenças, 16(2), 274-284.
Aquino, P. de S., Nicolau, A. I. O., \& Pinheiro, A. K. B. (2011). Desempenho das atividades de vida de prostitutas segundo o Modelo de Enfermagem de Roper, Logan e Tierney. Revista Brasileira de Enfermagem, 64(1), 136-144. Recuperado, de http:// www.scielo.br/scielo.php?script=sci_arttext\&pid $=$ S003471672011000100020\&lng $=$ en\&tlng $=$ pt

Ayres, J. R., Paiva, V., \& Buchalla, C. M. (2012). Direitos humanos e vulnerabilidade na prevenção e promoção da Saúde: Uma introdução. Em V. Paiva, J. R. Ayres, \& C. M. Buchalla (Eds.), Vulnerabilidade e Direitos Humanos. Prevenção e promoção da saúde. Da doença à cidadania (pp. 9-22). Curitiba: Juruá.

Brasil (2013). Ministério do Desenvolvimento Social e Combate à Fome. Relatórios de Informações Sociais da SAGI/MDS. Brasília: MDS. Recuperado de http://aplicacoes.mds.gov.br/sagi/RIv3/geral/ index.php.

Camargo, C. F., Curralero, R. B., Licio, E. C., \& Mostafa, J. (2013). Perfil socioeconômico dos beneficiários do Programa Bolsa Família: O que o cadastro único revela? Em T. Campello \& M. C. Neri. (Eds.). Programa Bolsa Família: uma década de inclusão e cidadania (pp. 157-177). Brasília: Ipea.

Camurça, C. E., Alencar, A., Cidade, E. \& Ximenes, V. (2016). Implicações psicossociais da seca na vida de moradores de um município da zona rural do nordeste do Brasil. Avances en Psicología Latinoamericana, 34(1), 117-128. doi: 10.12804/apl34.1.2016.08.

Carlotto, M. S., Amazarray, M. R., Chinazzo, Í., \& Taborda, L. (2011). Transtornos mentais comuns e fatores associados em trabalhadores: Uma análise na perspectiva de gênero. Caderno de Saúde Coletiva, 19 (2), 172-8. Recuperado de http://www.iesc. ufrj.br/cadernos/images/csc/2011_2/artigos/ csc_v19n2_172-178.pdf

Cidade, E. C., Moura Jr. J. F., \& Ximenes, V. M (2012). Implicações psicossociológicas da pobreza no povo latino-americano. Psicologia Argumento, 30(68), 87-98.

Costa, A. G. \& Ludermir, A. B. (2005). Transtornos mentais comuns e apoio social: Estudo em comunidade rural da Zona da Mata de Pernambuco, Brasil. Cad. Saúde Pública, 21(1), 73-79. Recuperado de http:// www.scielo.br/scielo.php?script $=$ sci_arttext\&pi$\mathrm{d}=$ S0102-311X2005000100009\&lng=en\&nrm $=$ iso 
Costa, M. G. S. G., Dimenstein, M., \& Leite, J. F. (2014). Condições de vida, gênero e saúde mental entre trabalhadoras rurais assentadas. Estudos de Psicologia (Natal), 19(2), 145-154.

Cotta, R. M. M., Gomes, A. P., M., Maia, T. M., Magalhães, K. A., Marques, E. S., \& Siqueira-Batista, R. (2007). Pobreza, injustiça, e desigualdade social: Repensando a formação de profissionais de saúde. Revista Brasileira de Educaşão Médica, 31(3), 278-286. doi: 10.1590/S0100-55022007000300010

Crespo, A. P. A. \& Gurovitz, E. (2002). A pobreza como um fenômeno multidimensional. $\mathrm{R} A$ E-eletrônica, 1(2), 1-12.

Detoni, P. P (2010). Seguir barragem: (RE-DES) Construções das masculinidades num canteiro de obras de uma usina hidrelétrica (Dissertação de mestrado). Universidade Federal do Rio Grande do Sul.

Ewald, A. P., Moura, M. T. C., \& Goulart, S. M. S (2012). Contemporaneidade e sofrimento psíquico: Relações entre modos de vida e demandas psicoterapêuticas. Psicologia Argumento, 30(68), 119-129.

Ferreira, L. N., Sales, Z. N., Casotti, C. A., Bispo Júnior, J. P., \& Braga Júnior, A. C. R. (2011). Perfil do consumo de bebidas alcoólicas e fatores associados em um município do Nordeste do Brasil. Cadernos de Saúde Pública, 27(8), 1473-1486.

Guimarães, M. B. L., Lima, C. M., Savi, E. A., Cardoso, E., Valla, V. V., Stotz, E. N., Lacerda, A., \& Santos, M. S. (2011). Os impasses da pobreza absoluta: A experiência da ouvidoria coletiva na região da Leopoldina, Rio de Janeiro (RJ, Brasil). Ciência \& Saúde Coletiva, 16(1), 291-300.

Instituto Nacional de Ciência e Tecnologia para Políticas Públicas do Álcool e outras Drogas [INPAD]. (2012). II Levantamento Nacional de Alcool e Outras Drogas - LENAD. São Paulo.

Instituto Nacional de Colonização e Reforma Agrária [INCRA] (2010). Pesquisa Sobre a Qualidade de Vida, Produção e Renda dos Assentamentos da Reforma Agrária Brasília: Autor.

Lindner, S. R., Coelho, E. B. S., Bolsoni, C. C., Rojas, P. F., \& Boing, A. F. (2015). Prevalência de violência física por parceiro íntimo em homens e mulheres de Florianópolis, Santa Catarina, Brasil: Estudo de base populacional. Cadernos de Saúde Pública, 31(4),815-826.
Loureiro, A., Lima, J., Partidário, M. R., \& Santana, P. (2015). Condicionantes da saúde mental e os instrumentos de avaliação de impactos. Em P. Santana (Eds.). Território e Saúde Mental em Tempos de Crise. (pp. 11-27). Coimbra: Universidade de Coimbra.

Maragno, L., Goldbaum, M., Gianini, R. J., Novaes, H. M. D., \& César, C. L. G. (2006). Prevalência de transtornos mentais comuns em populações atendidas pelo Programa Saúde da Família (QUALIS) no Município de São Paulo, Brasil. Cad. SaúdePública, 22 (8), 1638-1648.

Mari J. J., \& Williams P. (1986). A validity study of a psychiatric screening questionnaire (SRQ$20)$ in primary care in the city of São Paulo. $B r$ J Psychiatry, 148,23-26.

Martins, A. L. (2012). Investigando possiveis relações entre alcoolismo, sofrimento psíquico e violência intrafamiliar na perspectiva de mulheres atendidas pela Saúde da Família (Dissertação de mestrado). Universidade Estadual Paulista.

Mauriel, A. P. O. (2010). Capitalismo, politicas sociais e combate à pobreza. Ijuí: Ed. Ijuí.

Mello, J. (2015). A inclusão produtiva rural no Brasil sem miséria: $O$ desafio da superação da pobreza no campo. Caderno de Estudos Desenvolvimento Social em Debate (número 23). Brasília: Ministério do Desenvolvimento Social e Combate à Fome.

Miranda, M. P. M., Paula, C. S., \& Bordin, I. A. (2010). Violência conjugal física contra a mulher na vida: Prevalência e impacto imediato na saúde, trabalho e família. Revista Panamericana Salud Publica, 27(4), 300-308.

Moura, J. F; Ximenes, V. M., \& Sarriera, J. C. (2014). A construção opressora da pobreza no Brasil e suas consequências no psiquismo. Quaderns de Psicología, 16(2), 85-93.

Oliveira, L. Z., Cazella, A. A., Tecchio, A., \& Mireski, M. C. (2015). Ações da política de desenvolvimento territorial para superar a pobreza rural: Estudo de caso no território Meio Oeste Contestado (SC). Revista Brasileira de Gestão e Desenvolvimento Territorial, 11(1), 323-348.

Organização Mundial de Saúde (OMS). (2009). Integração de Saúde Mental nos cuidados de saúde primários: Uma perspectiva global. Recuperado de 
http:/ /www.who.int/eportuguese/publications/ Integracao_saude_mental_cuidados_primar ios.pdf

Organização Mundial de Saúde (OMS) (2014). Global status reporton alcohol and health - 2014. Geneva: WHO. Recuperado de http://apps.who.int/iris/ bitstream/10665/112736/1/9789240692763_eng. pdf?ua $=1$

Organización Panamericana de la Salud (2015). Informe sobre la situación regional sobre el alcoholy la salud em las Américas.Washington, DC: OPS.

Ottonelli, J., \& Mariano, J. L. (2014). Pobreza multidimensional nos municípios da Região Nordeste. Revista de Administração Pública, 48(5), 1253-1279.

Pereira, V. C. L. S., Andrade, F. A., Espínola, L. L., Azevedo, E. B., Nogueira, J. A., \& Ferreira Filha, M. O. (2015). Sofrimento psíquico em adolescentes associado ao alcoolismo familiar: Possíveis fatores de risco. Revista Eletrônica de Enfermagem, 17(2):178-85.

Pinho, P. S., \& Araújo, T. M. (2012). Associação entre sobrecarga doméstica e transtornos mentais comuns em mulheres. Revista Brasileira de Epidemiologia, 15(3), 560-572. Recuperado de http://www.scielo.br/ scielo.php?script $=$ sci_arttext\&pid $=\mathrm{S} 1415-790 \mathrm{X} 2$ 012000300010\&lng=en\&tlng=pt. 10.1590/ S1415-790X2012000300010

Portugal, F. B., Corrêa, M. A. P., \& Siqueira, M. M. (2010). Alcoolismo e comorbidade em um programa de assistência aos dependentes de álcool. SMAD. Revista eletrônica saúde mental álcool e drogas, 6(1), 1-13. Recuperado de http:// pepsic.bvsalud.org/scielo.php? script $=$ sci_ arttext\&pid=S1806-69762010000100006\&lng $=$ pt\& thng $=\mathrm{pt}$

Pussetti, C. \& Brazzabeni, M. (2011). Sofrimento social: Idiomas da exclusão e políticas do assistencialismo. Etnográfica, 15(3): 467-478.

Remoaldo, P. \& Nogueira, H. (2013). Variações e desigualdades socioterritoriais em saúde. Em P. Remoaldo \& H. Nogueira (Eds.), Desigualdades Socioterritoriais e Comportamentos em saúde (pp. 11-30) Lisboa: Colibri.
Sawaia, B. B. (2009). Psicologia e desigualdade social: Uma reflexão sobre liberdade e transformação social. Psicologia \& Sociedade, 21(3), 364-372.

Schneider, S. (2010). Situando o desenvolvimento rural no Brasil: O contexto e as questões em debate. Revista de Economia Política, 30(3), 511-531.

Silva, M., Cardoso, G., Saraceno, B., \& Almeida, J. C. (2015). Saúde mental e a crise econômica. Em P. Santana (Coord). Território e Saúde Mental em Tempos de Crise. (pp. 61-74). Universidade de Coimbra, PT.

Silva, R. A., \& Menezes, J. A. (2014). Mulheres jovens e suas vivências com o uso do álcool no contexto das comunidades quilombolas. Comunicação apresentada no $18^{\circ}$ Encontro da Rede Feminista Norte e Nordeste de Estudos e Pesquisas sobre a Mulher e Relações de Gênero, Recife. Resumo recuperado de http://www.ufpb.br/evento/lti/ocs/index. $\mathrm{php} / 18$ redor/18redor/paper/viewFile/732/785

Siqueira. L. (2013). Pobreza e serviço social: Diferentes concepções e compromissos políticos. São Paulo: Cortez.

Souza, P. H. G. F., \& Osorio, R. G. (2013). O perfil da pobreza no Brasil e suas mudanças entre 2003 e 2011. Em T. Campello \& M. C. Neri (Eds.), Programa Bolsa Família: uma década de inclusão e cidadania (pp. 139-155). Brasília: Ipea.

Spink, M. J. P, Brigadão, I. M, Nascimento, V. L. V, \& Cordeiro, M. P. (2014). A produção de informação na pesquisa social: Compartilhando ferramentas. Rio de Janeiro: Centro Edelstein de Pesquisas Sociais.

Vidal, C. E. L., Amara, B., Ferreira, D. P., Dias, I. M. F., Vilela, L. A. \& Franco, L. R. (2014). Preditores de prováveis transtornos mentais comuns (TMC) em prostitutas utilizando o Self Reporting Questionnaire. Jornal Brasileiro de Psiquiatria, 63(3), 205-212. doi: 10.1590/0047-2085000000027

Recebido em: 19/03/2016 Reformulado em: 01/05/2016; 21/05/2016 Aprovado em: 24/05/2016 
Sobre os autores:

Magda Dimenstein é professora titular da UFRN, graduada em Psicologia pela UFPE, mestre em Psicologia Clínica pela PUC/RJ e doutora em Saúde Mental pelo IPUB/UFRJ. Realizou estágio pós-doutoral na Universidad Alcalá de Henares - Espanha. Está vinculada ao PPGPSI/UFRN, é bolsista 1B do CNPq e atua na área de Saúde Coletiva com ênfase em Saúde Mental, Atenção Primária e Formação do Psicólogo. Endereço para correspondência: UFRN, CCHLA, Depto. de Psicologia, Campus Universitário, Lagoa Nova, Natal/RN, CEP: 59780-970.

E-mail:mgdimenstein@gmail.com

João Paulo Sales Macedo é professor adjunto da UFPI, doutor em Psicologia pela UFRN, está vinculado ao Programa de Pós-Graduação em Políticas Públicas/UFPI e atua na área da Saúde Coletiva e Saúde Mental, com foco na formação e prática do psicólogo nas políticas públicas e aspectos relacionados à interiorização da profissão.

E-mail: jampamacedo@gmail.com

Jader Leite é professor adjunto da UFRN, doutor em Psicologia pela UFRN, realizou estágio pós-doutoral junto ao Núcleo de Psicologia Comunitária (NUCOM) da UFC e está vinculado ao PPGPsi/UFRN. Atua, ainda, no campo da Psicologia social a partir dos temas: movimentos sociais e contextos rurais, relações de gênero, saúde mental e processos de subjetivação.

E-mail:jaderfleite@gmail.com

Candida Dantas é professora adjunta da UFRN, doutora em Psicologia pela UFRN e possui experiência acadêmica e de pesquisa em Psicologia, atuando nos seguintes temas: formação e atuação do psicólogo, história da psicologia, relações de gênero e pobreza.

E-mail: candida.dantas@gmail.com

Monique Pfeifer Rodrigues da Silva é graduanda em Psicologia na Universidade Federal do Rio Grande do Norte (UFRN), bolsista de PIBIC/IC e formada em geologia e Mineração pelo Instituto Federal de Educação, Ciência e Tecnologia do Rio Grande do Norte (IFRN) na modalidade técnico integrado ao Ensino Médio.

E-mail: niq.pfeifer@hotmail.com

Contato com os autores:

UFRN, CCHLA, Depto. de Psicologia

Programa de Pós-Graduação em Psicologia/PPGPSi

Campus Universitário - Lagoa Nova

Natal-RN, Brasil

CEP: 59078-970

E-mail:magda@ufrnet.br 
